\title{
Simultaneous detection of the nonlinear restoring and excitation of a forced nonlinear oscillation: An integral approach
}

\author{
Fayyaz Ahmad ${ }^{\mathrm{a}}$, Taek Soo Jang ${ }^{\mathrm{b}, *}$, Jinsoo Park ${ }^{\mathrm{b}}$, Hong Gun Sung ${ }^{\mathrm{c}}$ \\ ${ }^{a}$ Department de Física i Enginyeria Nuclear, universitat Politècnica de Catalunya, Comte d'Urgell 187, 08036 Barcelona, Spain \\ ${ }^{b}$ Department of Naval Architecture and Ocean Engineering, Pusan National University, Busan 609-735, Republic of Korea \\ ${ }^{c}$ Korea Research Institute of Ships and Ocean Engineering (KRISO; previously MOERI or MOERI/KIOST), Daejeon 305-343, Republic of Korea
}

\begin{abstract}
We address in this article, how to calculate the restoring characteristic and the excitation of a nonlinear forced oscillating system. Under the assumption that the forced nonlinear oscillator has a periodic solution with period $T=2 \pi / \omega$, we constructed a system of linear equations by introducing time-dependent multipliers. The periodicity assumption helps to simplify the system of linear equations. The stability and uniqueness are also presented for the inverse problem. Numerical testing is conducted to show the effectiveness of our presented methodology.
\end{abstract}

Keywords: Forced nonlinear oscillator, Inverse problem, Periodic solution, Nonlinear restoring and excitation

\section{Introduction}

Known as the art and science, system identification is of building mathematical models of physical systems from observed data, which has been of great significance in mathematical sciences as well as applied engineering. Especially in engineering applications, the issue of identifying a forced nonlinear dynamic system, being involved in the present work, appears to be fascinating and significant, because its understanding is of great importance for the practical design of nonlinear systems in real world. From application viewpoint, the nonlinear characteristics of a ship motion can be modeled using Duffing equation and hence their identification from experimentally measured data is an interesting area of study. Even though, there have been a lot of valuable works regarding the identification of forced systems, most studies of them are limited to only the force identification that recovers external single force excitations. For example, Doyle [1] proposed a wavelet-based deconvolution method for recovering impact forces of a system. From measurements, a method of identification of system parameters and sinusoidal and impulsive forces was suggested by $\mathrm{Lu}$ and Law [2]. A new mathematical procedure was proposed for measuring non-harmonic periodic excitation forces in nonlinear damped systems( Jang et al. [3]).

However, in contrast to the previous (single) force identification, this paper concerns a simultaneous identification, recovering not only the force excitations but the restoring characteristic of a forced nonlinear system. In fact, little studies have been tried for the identification of both the force excitations and the restoring characteristic in forced nonlinear systems: that is, most of progress has been made on the (single) identification of either or force excitations or restoring characteristic.

In a recent article [4], Jang proposed a mathematical process for recovering the external excitation, as well as the linear and nonlinear restoring force coefficients of a forced nonlinear oscillator. He, based on introducing J-function and its zero-crossing, used the energy balance idea to estimate the value of external excitation under assumption that nonlinear oscillator has a periodic solution. The detection of time-varying nonlinear damping in nonlinear oscillation systems is explored in [5] with the help of integral equation formulation. The idea of zero-crossing is again presented in [6] to recover amplitude and nonlinear restoring coefficients. In [7], the simultaneous detection of nonlinear damping,

\footnotetext{
${ }^{*}$ Corresponding author

Email addresses: fayyaz.ahmad@upc.edu (Fayyaz Ahmad), taek@pusan.ac.kr (Taek Soo Jang ), jinsu@pusan.ac.kr (Jinsoo Park ), Email of Dr. Hong Gun Sung is needed (Hong Gun Sung)
} 
phase shift and amplitude of external harmonic excitation is studied. The designed problem was ill-posed, and a regularization scheme is used to get faithful estimation of parameters. The nonlinear oscillators are well studied by many researchers for instant see [8-16] and references therein. A valuable investigation has been conducted to detect the parameters from noisy data for forced nonlinear oscillators[17-24] and provides base for the new exploration in area of inverse problems.

In the present study we extend the idea which was presented in [4] for the detection of amplitude by introducing a set of continuous functions $\psi_{j}$. In this paper, based on the idea of [4], we would like to present a new methodology with an integral approach. We construct a system of linear equations by introducing a set of continuous multipliers. Thereby, a general procedure will be provided for calculating the two restoring coefficients and the external force. Finally, the uniqueness and the stability are also investigated and numerical experiments are given, showing that our presented methodology is efficient and accurate.

\section{Inverse problem for forced nonlinear oscillator}

The nonlinear forced oscillator, under the action of an external harmonic load $\Gamma \cos (\omega t)$ with amplitude $\Gamma>0$ and frequency $\omega>0$, can be written as

$$
m \ddot{q}+\phi(\dot{q})+k_{1} q+k_{2} q^{3}=\Gamma \cos (\omega t),
$$

where the constant $m>0$ stands for mass of a particle of an oscillator and $\phi(\dot{q})$ is the nonlinear damping force. The linear and nonlinear restoring constants are denoted by $k_{1}$ and $k_{2}$ respectively in (1). We make assumption that oscillating system exhibits periodic solution with period $T(=2 \pi / \omega)$ i.e.

$$
q(t)=q(t+T), \quad \text { as } t \rightarrow \infty .
$$

In practice, we have measurements for $q(t)$ and $\dot{q}(t)$ with some noise and the goal is to restore $k_{1}, k_{2}$ and $\Gamma$.

\section{Formulation of inverse problem}

The Eq. (1) can be written as

$$
q k_{1}+q^{3} k_{2}-\cos (\omega t) \Gamma=-m \ddot{q}-\phi(\dot{q}) .
$$

Now we consider functions $\psi_{i} \equiv \psi_{i}(t, q, \dot{q})$ for $i \in\{1,2,3\}$ which are continuous with respect to all its arguments. We construct system of three linear equations by using (3) and $\psi_{i}$ as follows

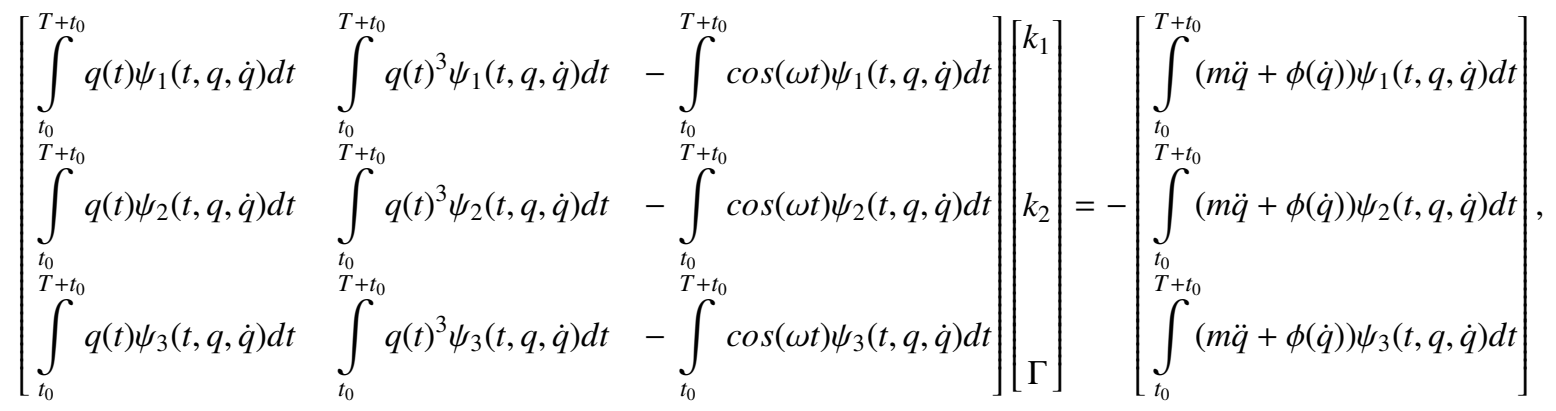

where $t_{0} \rightarrow \infty$.

\section{Uniqueness of solution}

We select $\psi_{i}$ in a way that the determinant of the matrix on the left-hand side of Eq. 4 is non-zero. Clearly, the nonzero determinant gives us unique solution for system of linear equations for selected set of $\psi_{i}$. 


\section{Stability of solution}

Let $\left\{q_{n}(t)\right\}$ be a sequence such that $\lim _{n \rightarrow \infty} q_{n}(t)=q(t)$ and

$$
\begin{aligned}
& I_{i, 1}(q, \dot{q})=\lim _{t_{0} \rightarrow \infty} \int_{t_{0}}^{T+t_{0}} q(t) \psi_{i}(t, q, \dot{q}) d t, \\
& I_{i, 2}(q, \dot{q})=\lim _{t_{0} \rightarrow \infty} \int_{t_{0}}^{T+t_{0}} q(t)^{3} \psi_{i}(t, q, \dot{q}) d t, \\
& I_{i, 3}(q, \dot{q})=\lim _{t_{0} \rightarrow \infty} \int_{t_{0}}^{T+t_{0}} \cos (\omega t) \psi_{i}(t, q, \dot{q}) d t .
\end{aligned}
$$

The continuity of integral and $\psi_{i}(t, q, \dot{q})$ produce the following results

$$
\begin{aligned}
& \lim _{n \rightarrow \infty} I_{i, 1}\left(q_{n}, \dot{q}_{n}\right)=\lim _{\substack{t_{0} \rightarrow \infty \\
n \rightarrow \infty}} \int_{t_{0}}^{T+t_{0}} q_{n}(t) \psi_{i}\left(t, q_{n}, \dot{q}_{n}\right) d t=\lim _{t_{0} \rightarrow \infty} \int_{t_{0}}^{T+t_{0}} \lim _{n \rightarrow \infty} q_{n}(t) \psi_{i}\left(t, \lim _{n \rightarrow \infty} q_{n}, \lim _{n \rightarrow \infty} \dot{q}_{n}\right) d t, \\
& \lim _{n \rightarrow \infty} I_{i, 2}\left(q_{n}, \dot{q}_{n}\right)=\lim _{\substack{t_{0} \rightarrow \infty \\
n \rightarrow \infty}} \int_{t_{0}}^{T+t_{0}} q_{n}(t)^{3} \psi_{i}\left(t, q_{n}, \dot{q}_{n}\right) d t=\lim _{t_{0} \rightarrow \infty} \int_{t_{0}}^{T+t_{0}}\left(\lim _{n \rightarrow \infty} q_{n}(t)\right)^{3} \psi_{i}\left(t, \lim _{n \rightarrow \infty} q_{n}, \lim _{n \rightarrow \infty} \dot{q}_{n}\right) d t, \\
& \lim _{n \rightarrow \infty} I_{i, 3}\left(q_{n}, \dot{q}_{n}\right)=\lim _{\substack{t_{0} \rightarrow \infty \\
n \rightarrow \infty}} \int_{t_{0}}^{T+t_{0}} \cos (\omega t) \psi_{i}\left(t, q_{n}, \dot{q}_{n}\right) d t=\lim _{t_{0} \rightarrow \infty} \int_{t_{0}}^{T+t_{0}} \cos (\omega t) \psi_{i}\left(t, \lim _{n \rightarrow \infty} q_{n}, \lim _{n \rightarrow \infty} \dot{q}_{n}\right) d t
\end{aligned}
$$

From Eqs. 5 we have

$$
\begin{aligned}
& \lim _{n \rightarrow \infty} I_{i, 1}\left(q_{n}, \dot{q}_{n}\right)=I_{i, 1}\left(\lim _{n \rightarrow \infty} q_{n}, \lim _{n \rightarrow \infty} \dot{q}_{n}\right), \\
& \lim _{n \rightarrow \infty} I_{i, 2}\left(q_{n}, \dot{q}_{n}\right)=I_{i, 2}\left(\lim _{n \rightarrow \infty} q_{n}, \lim _{n \rightarrow \infty} \dot{q}_{n}\right), \\
& \lim _{n \rightarrow \infty} I_{i, 3}\left(q_{n}, \dot{q}_{n}\right)=I_{i, 3}\left(\lim _{n \rightarrow \infty} q_{n}, \lim _{n \rightarrow \infty} \dot{q}_{n}\right) .
\end{aligned}
$$

With similar reasoning, one can obtain

$$
\begin{aligned}
& \lim _{\substack{t_{0} \rightarrow \infty \\
n \rightarrow \infty}} \int_{t_{0}}^{T+t_{0}}\left(m \ddot{q}_{n}+\phi\left(\dot{q}_{n}\right)\right) \psi_{1}(t, q, \dot{q}) d t=\lim _{t_{0} \rightarrow \infty} \int_{t_{0}}^{T+t_{0}}\left(m \lim _{n \rightarrow \infty} \ddot{q}_{n}+\phi\left(\lim _{n \rightarrow \infty} \dot{q}_{n}\right)\right) \psi_{1}\left(t, \lim _{n \rightarrow \infty} q_{n}, \lim _{n \rightarrow \infty} \dot{q}_{n}\right) d t, \\
& \lim _{\substack{t_{0} \rightarrow \infty \\
n \rightarrow \infty}} \int_{t_{0}}^{T+t_{0}}\left(m \ddot{q}_{n}+\phi\left(\dot{q}_{n}\right)\right) \psi_{2}(t, q, \dot{q}) d t=\lim _{t_{0} \rightarrow \infty} \int_{t_{0}}^{T+t_{0}}\left(m \lim _{n \rightarrow \infty} \ddot{q}_{n}+\phi\left(\lim _{n \rightarrow \infty} \dot{q}_{n}\right)\right) \psi_{2}\left(t, \lim _{n \rightarrow \infty} q_{n}, \lim _{n \rightarrow \infty} \dot{q}_{n}\right) d t, \\
& \lim _{\substack{t_{0} \rightarrow \infty \\
n \rightarrow \infty}} \int_{t_{0}}^{T+t_{0}}\left(m \ddot{q}_{n}+\phi\left(\dot{q}_{n}\right)\right) \psi_{3}(t, q, \dot{q}) d t=\lim _{t_{0} \rightarrow \infty} \int_{t_{0}}^{T+t_{0}}\left(m \lim _{n \rightarrow \infty} \ddot{q}_{n}+\phi\left(\lim _{n \rightarrow \infty} \dot{q}_{n}\right)\right) \psi_{3}\left(t, \lim _{n \rightarrow \infty} q_{n}, \lim _{n \rightarrow \infty} \dot{q}_{n}\right) d t
\end{aligned}
$$

The Eq. 4 can be recast as

$$
\begin{aligned}
& \mathbf{A}(q, \dot{q}) \mathbf{x}(q, \dot{q})=\mathbf{b}(q, \dot{q}), \\
& \mathbf{x}(q, \dot{q})=\mathbf{A}(q, \dot{q})^{-1} \mathbf{b}(q, \dot{q}),
\end{aligned}
$$


where $\mathbf{A}$ is the matrix and $\mathbf{b}$ is the vector on the right-hand side in Eqs. 4 . The parameters $k_{1}, k_{2}, \Gamma$ vector is denoted by x. The Eqs. 6, 7 and 8 collectively conclude that

$$
\lim _{n \rightarrow \infty} \mathbf{x}\left(q_{n}, \dot{q}_{n}\right)=\mathbf{x}\left(\lim _{n \rightarrow \infty} q_{n}, \lim _{n \rightarrow \infty} \dot{q}_{n}\right),
$$

which means that the solution vector continuously depends on $q$ and $\dot{q}$ and stability is the consequence of continuous dependence.

\section{Calculation of parameters $k_{1}, k_{2}, \Gamma$}

Our presented methodology is very general because the selection of continuous $\psi_{i}$ have only one constrained i.e., $\operatorname{det}(\mathbf{A}) \neq 0$. We make the following selections for $\psi_{i}$

$$
\psi_{1}=q, \quad \psi_{2}=q^{3}, \quad \psi_{3}=\dot{q}
$$

The further implications of Eq. 2 gives us

$$
\begin{aligned}
& \lim _{t_{0} \rightarrow \infty} q^{s}\left(t_{0}+T\right)=\left.\lim _{t_{0} \rightarrow \infty} q^{s}\left(t_{0}\right) \Rightarrow \lim _{t_{0} \rightarrow \infty} q^{s}(t)\right|_{t_{0}} ^{t_{0}+T}=0, \\
& \lim _{t_{0} \rightarrow \infty} \dot{q}^{s}\left(t_{0}+T\right)=\left.\lim _{t_{0} \rightarrow \infty} \dot{q}^{s}\left(t_{0}\right) \Rightarrow \lim _{t_{0} \rightarrow \infty} \dot{q}^{s}(t)\right|_{t_{0}} ^{t_{0}+T}=0, \\
& \left.\lim _{t_{0} \rightarrow \infty}\{\sin (\omega t) \text { or } \cos (\omega t)\} q^{s}(t)\right|_{t_{0}} ^{t_{0}+T}=0,
\end{aligned}
$$

where $s$ is a positive integer. By using (10), we produced the following useful relationships

$$
\begin{aligned}
& \lim _{t_{0} \rightarrow \infty} \int_{t_{0}}^{T+t_{0}} q^{s}(t) \ddot{q} d t=-s \lim _{t_{0} \rightarrow \infty} \int_{t_{0}}^{T+t_{0}} q^{s-1}(t) \dot{q}^{2} d t, \quad \lim _{t_{0} \rightarrow \infty} \int_{t_{0}}^{T+t_{0}} q^{s}(t) \dot{q}(t) d t=0, \quad \lim _{t_{0} \rightarrow \infty} \int_{t_{0}}^{T+t_{0}} \dot{q}(t) \ddot{q}(t) d t=0, \\
& \lim _{t_{0} \rightarrow \infty} \int_{t_{0}}^{T+t_{0}} \cos (\omega t) q^{s}(t) d t=-\frac{s}{\omega} \lim _{t_{0} \rightarrow \infty} \int_{t_{0}}^{T+t_{0}} \sin (\omega t) q^{s-1}(t) \dot{q} d t, \quad \lim _{t_{0} \rightarrow \infty} \int_{t_{0}}^{T+t_{0}} \cos (\omega t) \dot{q}(t) d t=\omega \lim _{t_{0} \rightarrow \infty} \int_{t_{0}}^{T+t_{0}} \sin (\omega t) q(t) d t .
\end{aligned}
$$

The substitution of (9) in (4) gives

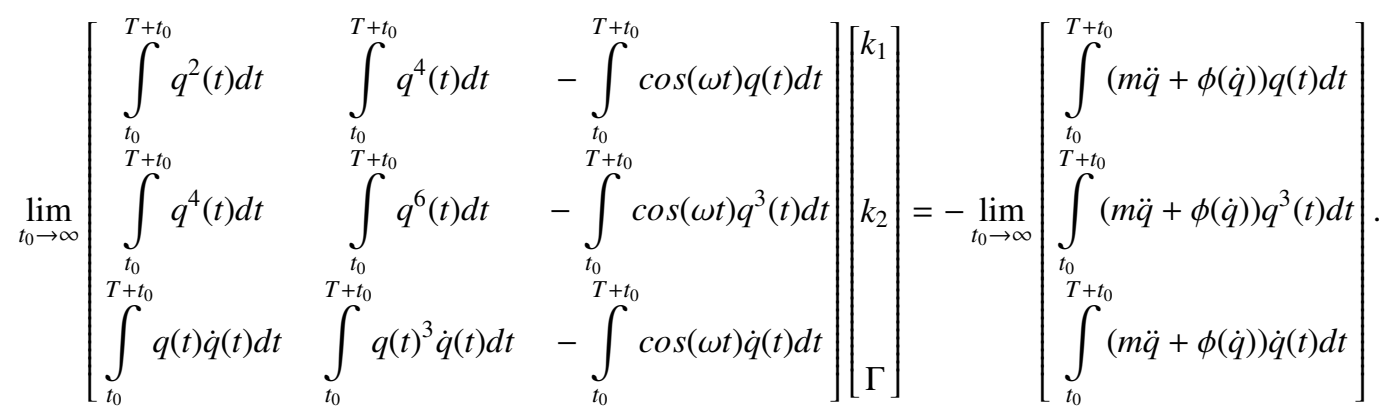


The relationships in (11) further simplify (12)

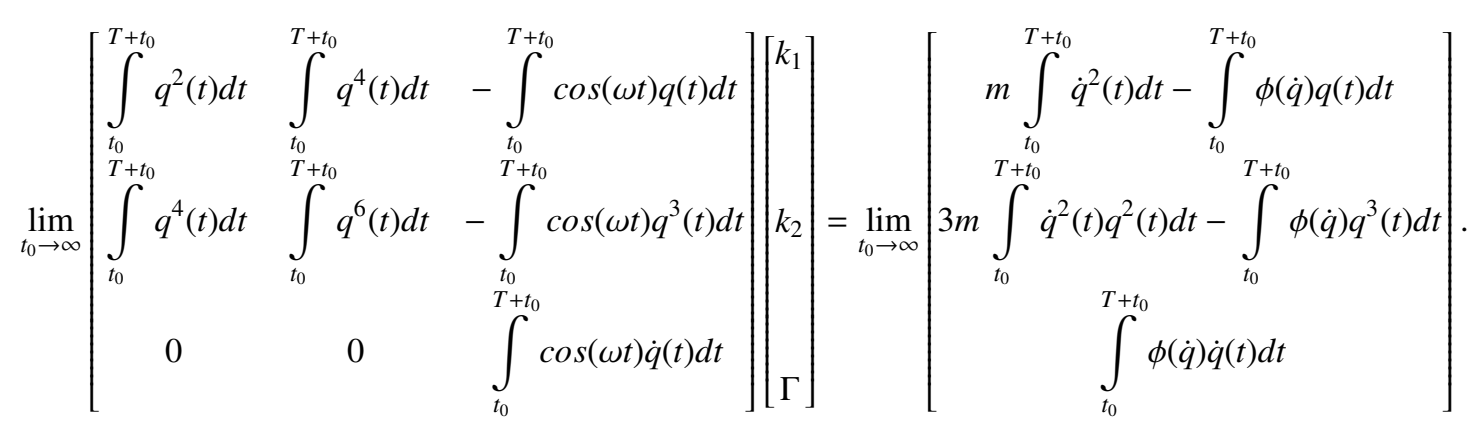

The alternative expression for (13) is

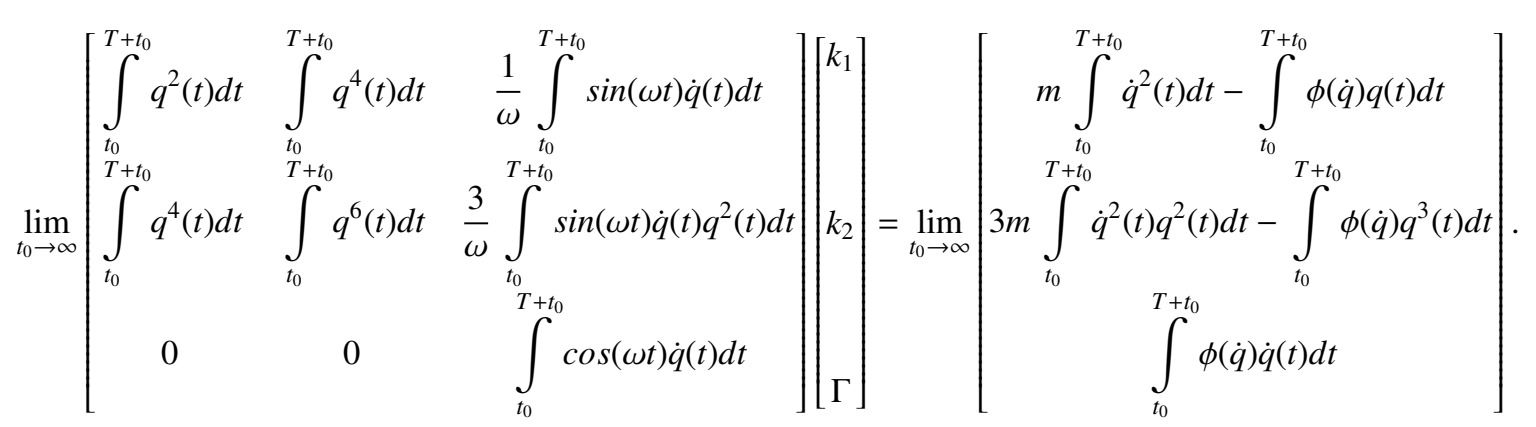

The third equation in (14) provides the value of $\Gamma$

$$
\Gamma=\lim _{t_{0} \rightarrow \infty} \frac{\int_{t_{0}}^{T+t_{0}} \phi(\dot{q}) \dot{q}(t) d t}{\int_{t_{0}}^{T+t_{0}} \cos (\omega t) \dot{q}(t) d t} \quad \text { or } \quad \Gamma=\lim _{t_{0} \rightarrow \infty} \frac{\int_{t_{0}}^{T+t_{0}} \phi(\dot{q}) \dot{q}(t) d t}{\omega \int_{t_{0}}^{T+t_{0}} \sin (\omega t) q(t) d t} .
$$

In both values of $\Gamma$ the denominator should be non-zero in (15). One of the alternative expressions for $\psi_{i}$ could be $a_{i} q+b_{i} q^{3}+c_{i} \dot{q}$. The selection of odd powers of $q$ in the definition of $\psi_{i}$ provide better numerical approximated values of parameters. The presented methodology is novel because periodicity assumption only helps to simplify the integrals and selection of $\psi_{i}$ are also not very restricted. Moreover, the developed methodology can also be used to restore parameters in multi-degree of freedom systems for instance in Duffing equation with two degrees of freedom.

\section{Numerical testing}

In this section, we conduct two numerical tests for two different cases of the nonlinear oscillator (1). The objective is to recover the coefficients of restoring and amplitude of external excitation from noisy measurements. The perturbed data can be obtained numerically by adding white noise to the noise-free data of displacements and velocities. The noise level and percentage error are defined as

$$
\begin{aligned}
& \delta=\frac{\left\|q_{\text {response }}-q^{\delta}\right\|_{2}}{\left\|q_{\text {response }}\right\|_{2}} \times 100(\%), \\
& \text { Error }(\%)=\frac{\mid \text { Exact value of parameter }- \text { Numerically computed value of parameter } \mid}{\mid \text { Exact value of parameter } \mid} \times 100
\end{aligned}
$$




\subsection{Case I}

For first case we define the damping law as $\phi(\dot{q})=c \dot{q}$. Then the first equation can be stated as

$$
m \ddot{q}+c \dot{q}+k_{1} q+k_{2} q^{3}=\Gamma \cos (\omega t)
$$

and consequently (13) gets the form

$$
\lim _{t_{0} \rightarrow \infty}\left[\begin{array}{ccc}
\int_{t_{0}}^{T+t_{0}} q^{2}(t) d t & \int_{t_{0}}^{T+t_{0}} q^{4}(t) d t & -\int_{t_{0}}^{T+t_{0}} \cos (\omega t) q(t) d t \\
\int_{t_{0}}^{T+t_{0}} q^{4}(t) d t & \int_{t_{0}}^{T+t_{0}} q^{6}(t) d t & -\int_{t_{0}}^{T+t_{0}} \cos (\omega t) q^{3}(t) d t \\
0 & \int_{t_{0}}^{T+t_{0}} \cos (\omega t) \dot{q}(t) d t
\end{array}\right]\left[\begin{array}{c}
k_{1} \\
m \int_{t_{0}}^{T+t_{0}} \dot{q}^{2}(t) d t \\
k_{2} \\
\int_{t_{0}}^{T+t_{0}} \\
3 m \int_{t_{0} \rightarrow \infty}^{T+t_{0}} \dot{q}^{2}(t) \dot{q}^{2}(t) d t
\end{array}\right] .
$$

The Eq. (16) is integrated up to $23 T$ to get the numerical data and the Figure 1 shows the profiles of displacement and velocity. The Figure 2 tells the evolution of displacement and velocity for a period $T$ and Figure 3 depicts the phase diagrams of closed orbits as well as white-noise effected closed orbit. In order to capture the real effect of white-noise on the restoring coefficients and amplitude, we run the simulations 2000 times for each noise level. We list maximum, minimum, mean percentage errors with respect to different noise levels in the Table 1. The maximum percentage error in all parameters is approximately equal to Mean $+5 \sigma$, here $\sigma$ is the standard deviation. The error in displacement and velocity profiles generated from the numerically recovered parameters (without white-noise) is plotted in Figure 4 . The increase in noise level enhances the error in the recovered values of restoring coefficients as well as that of in amplitude. The percentage error in linear coefficient is higher in comparison with amplitude and nonlinear coefficient in the first case. The recovered amplitude has less standard deviation and hence gives a best estimate for actual excitation.

\subsection{Case II}

In the present case, we use the same damping force model but the simulations are performed in different setting of parameters. The Figures 5, 6, 7 shed light on various aspects of simulations for the second case. The numerical results in Table 2 are in good agreement with that of Table 1 in the sense that the maximum percentage errors are approximately equal to Mean $+5 \sigma$. Again the percentage error in the linear coefficient is higher compare with other parameters. The estimated value of external excitation is more accurate and stable because it has less error in a mean value as well in standard deviation.Simulation values of $k_{1}, k_{2}$ and $\Gamma$ are plotted in Figures 8,9 and 10 respectively. It is clearly evident to get realistic estimates of parameters, we required suitable number of data sets of observed values of displacements and velocities.

Our analysis reveals that the presented methodology provides reasonable estimates for the restoring coefficients as well as for the amplitude. Our proposal is very general in the sense that we have freedom in the selection of continuous multipliers $\psi(t, q, \dot{q})$ for the integration of nonlinear oscillator over a single period of time to get a system of linear equations which in turns provide the good estimates of parameters. In the present study, we only explore a single choice for $\psi$ but further investigation can be carried out for the best selection of $\psi(t, q, \dot{q})$.

\section{Conclusions}

The presented methodology provides a very general method for the estimation of restoring coefficients and external excitation by selecting different continuous multipliers $\psi(t, q, \dot{q})$. The periodicity assumption helps to simplify the resulting system of a linear equation with parameters as unknown quantities. The developed formalism is a well-posed problem and does not face numerical instabilities. The maximum percentage error falls under Mean $+5 \sigma$ for all parameters in the present study to recover parameters from noisy data. 


\section{Acknowledgment}

This work was supported by a 2-Year Research Grant of Pusan National University.

\section{References}

[1] J. Doyle, A wavelet deconvolution method for impact force identification. Experimenal Mechanics 37(4) (1997) 403-408.

[2] Z.R. Lu, S.S. Law, Identification of system parameters and input force from output only. Mechanical Systems and Signal Processing 21(5) (2007) 2099-2111.

[3] T.S. Jang, Hyoungsu Baek, Hang S. Choi, Sun-Gu Lee, A new method for measuring nonharmonic periodic excitation forces in nonlinear damped systems, Mechanical Systems and Signal Processing 25 (6) (2011) 2219-2228.

[4] T.S. Jang, Uniqueness and stability of the simultaneous detection of the nonlinear restoring and excitation of a forced nonlinear oscillation, Applied Mathematics and Computation 228 (2014) 234-239.

[5] T. S. Jang, Hyoungsu Baek, M. C. Kim, B. Y. Moon, A new method for detecting the time-varying nonlinear damping in nonlinear oscillation systems: non-parametric identification, Mathematical Problems in Engineering Volume 2011, Article ID 749309, 12 pages, doi: $10.1155 / 2011 / 749309$

[6] T. S. Jang, A new simultaneous identification of the harmonic excitations and nonlinear damping of forced damped nonlinear oscillations: a parametric approach, Journal of Applied Mathematics Volume 2013, Article ID 754576, 7 pages http://dx.doi.org/10.1155/2013/754576

[7] T.S. Jang, A method for simultaneous identification of the full nonlinear damping and the phase shift and amplitude of the external harmonic excitation in a forced nonlinear oscillator, Computers and Structures 2013 (120-15) pp. 77-85.

[8] U. an der Heiden, A. Longtin, M. C. Maekey, J. G. Milton, R. Seholl, Oscillatory modes in a nonlinear second-order differential equation with delay, Journal of Dynamics and Differential Equations, Vol. 2, No. 4, 1990.

[9] A.Y.T. Leung, H.X. Yang, P. Zhu, Z.J. Guo, Steady state response of fractionally damped nonlinear viscoelastic arches by residue harmonic homotopy, Computers and Structures 121 (2013) 10-21.

[10] A.Y.T. Leung, Zhongjin Guo, H.X. Yang, Fractional derivative and time delay damper characteristics in Duffingvan der Pol oscillators, Commun Nonlinear Sci Numer Simulat 18 (2013) 2900-2915.

[11] Hong-guang Li, Guang Meng, Nonlinear dynamics of a SDOF oscillator with BoucWen hysteresis, Chaos, Solitons and Fractals 34 (2007) 337-343.

[12] S. L. Han and Takeshi Kinoshita, Stochastic inverse identification of nonlinear roll damping moment of a ship moving at nonzero-forward speeds, Mathematical Problems in Engineering Volume 2012, Article ID 769385, 22 pages doi:10.1155/2012/769385

[13] Wang Shimin, Yang Lechang, An analytical approximation method for strongly nonlinear oscillators, Journal of Applied Mathematics Volume 2012, Article ID 958121, 9 pages doi:10.1155/2012/958121

[14] A. Beledez , E. Gimeno , M. L. Alvarez , M. S. Yebra, D. I. Mendez, Analytical approximate solutions for conservative nonlinear oscillators by modified rational harmonic balance method, International Journal of Computer Mathematics, 87:7, 1497-1511, DOI: $10.1080 / 00207160802380942$

[15] T. A. Nofel, Application of the homotopy perturbation method to nonlinear heat conduction and fractional Van der Pol damped nonlinear oscillator, Applied Mathematics, 2014, 5, 852-861

[16] Yong-Jin Han, Dynamics of coupled nonlinear oscillators of different attractors; Van der Pol oscillator and damped Duffing oscillator, Journal of the Korean Physical Society, Vol. 37, No. 1, July 2000, pp. 3-9

[17] T.S. Jang, A new mathematical procedure for simultaneous identification of the nonlinear damping and restoring characteristics based on acceleration measurements, Ships and Offshore Structures, DOI:10.1080/17445302.2014.942084

[18] T.S. Jang, A novel method for the non-parametric identification of nonlinear restoringforces in nonlinear vibrations based on response data: a dissipative nonlinear dynamical system, Ships and Offshore Structures 2011 (6-4) pp.257-263.

[19] T.S. Jang, Non-parametric simultaneous identification of both the nonlinear damping and restoring characteristics of nonlinear systems whose dampings depend on velocity alone, Mechanical Systems and Signal Processing 2011 (25-4) pp.1159-1173.

[20] T.S. Jang, S.H. Kwon, J.H. Lee, Recovering the functional form of the nonlinear roll damping of ships from a free-roll decay experiment: an inverse formulism, Ocean Engineering 2010 (37-14,15) pp.1337-1344.

[21] T.S. Jang, H.S. Back, S.L. Han, T. Kinoshita, Indirect measurement of the impulsive load to a nonlinear system from dynamic responses: inverse problem formulation, Mechanical Systems and Signal Processing 2010 (24-6) pp.1665-1681.

[22] T.S. Jang, S.L. Han, T. Kinoshita, An inverse measurement of the sudden underwater movement of the sea-floor by using the time-history record of the water-wave elevation, Wave Motion 2010 (47-3) pp.146-155.

[23] T.S. Jang, S.H. Kwon, S.L. Han, A novel method for non-parametric identification of nonlinear restoring forces in nonlinear vibrations from noisy response data: a conservative system, Journal of Mechanical Science and Technology 2009 (23-11) pp.2938-2947.

[24] T.S. Jang, H.S. Choi, S.L. Han, A new method for detecting nonlinear damping and restoring forces in nonlinear oscillation systems from transient data, International Journal of Non-linear Mechanics 2009(44-7), pp. 801-808. 

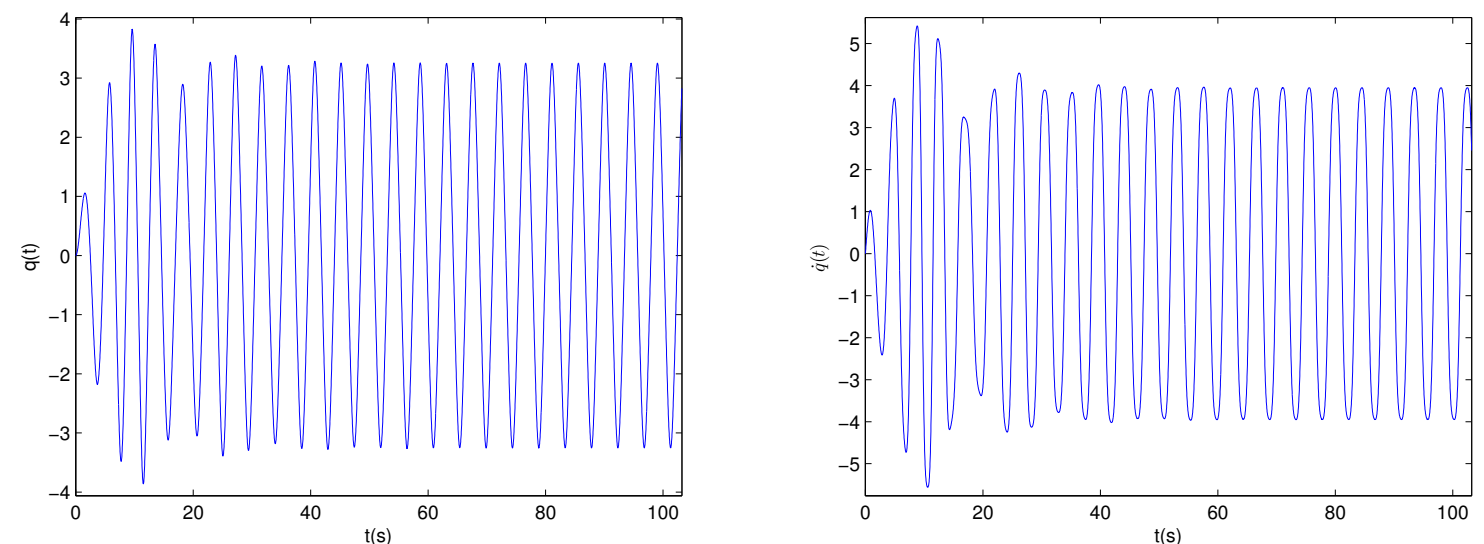

Figure 1: Case 1: displacement versus time(left), velocity versus time(right), $m=1, c=0.2, \omega=1.4, t_{0}=21 T, t=[0,23 T], d t=0.02$, $q(0)=\dot{q}(0)=0$
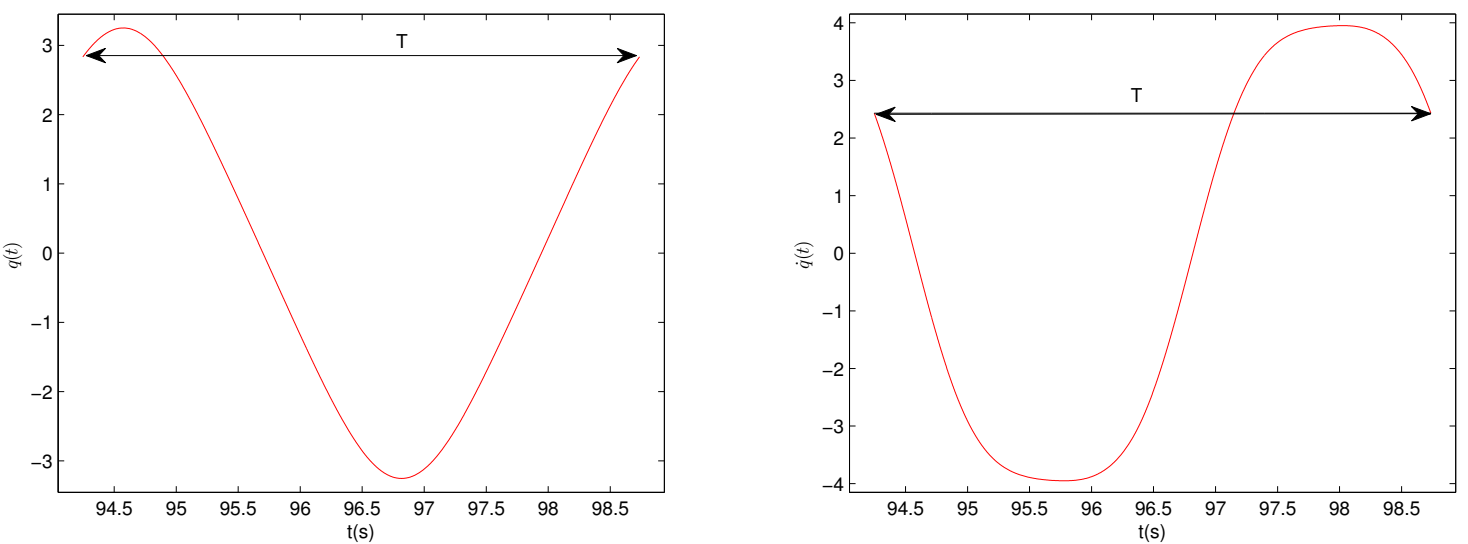

Figure 2: Case 1: displacement versus time(left), velocity versus time(right), $m=1, c=0.2, \omega=1.4, t_{0}=21 T, t=[0,23 T], d t=0.02$, $q(0)=\dot{q}(0)=0$
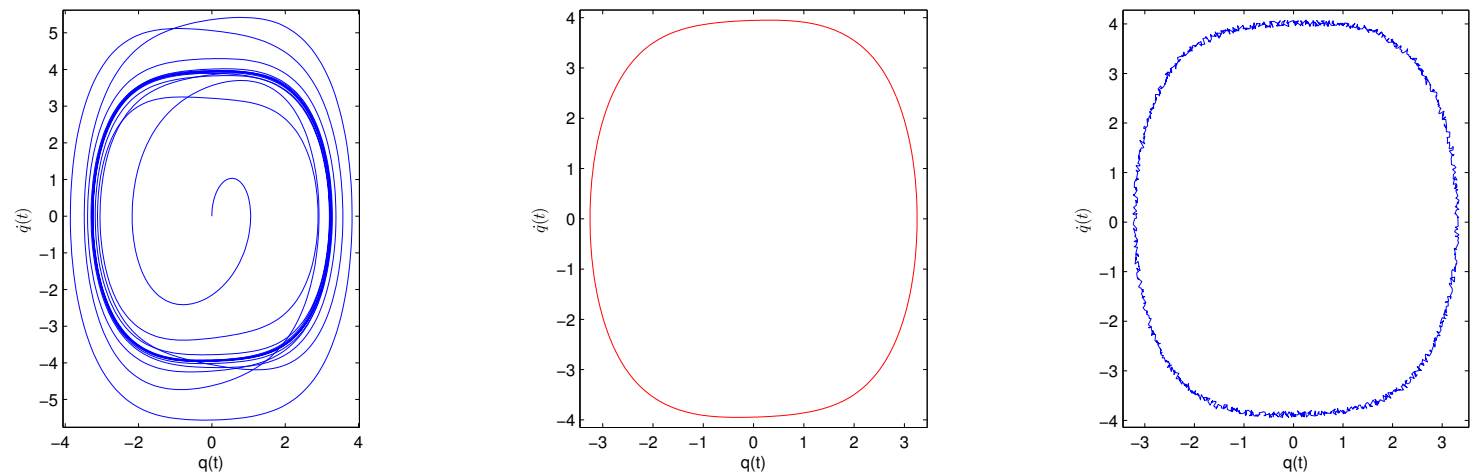

Figure 3: Case 1: orbits in phase space(left portrait convergence to closed orbits, center portrait without white noise, right portrait contaminated with $2.5 \%$ white noise), $m=1, c=0.2, \omega=1.4, t_{0}=21 T, t=[0,23 T], d t=0.02, q(0)=\dot{q}(0)=0$ 

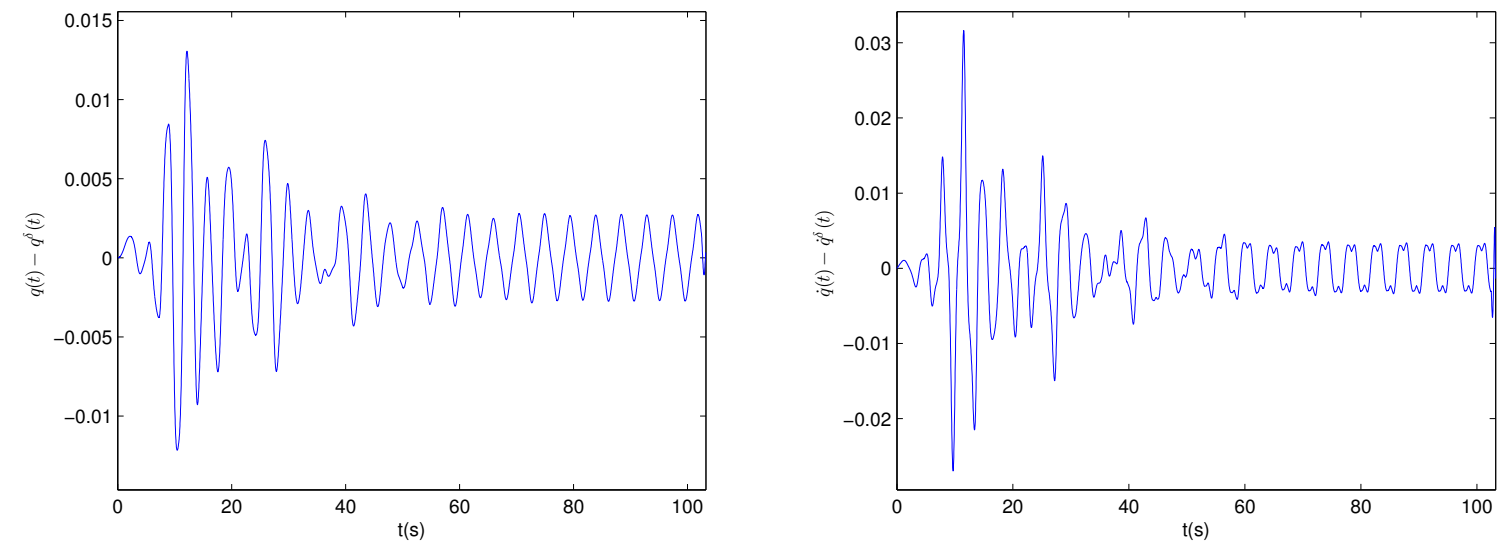

Figure 4: Case 1: Error plot of $q(t)$ and $\dot{q}(t)$ when percentage errors in $\Gamma, k_{1}, k_{2}$ are $5.4932 \times 10^{-2}, 2.1318 \times 10^{-1}, 2.9335 \times 10^{-1}$ respectively, $m=1$, $c=0.2, \omega=1.4, t_{0}=21 T, t=[0,23 T], d t=0.02, q(0)=\dot{q}(0)=0$
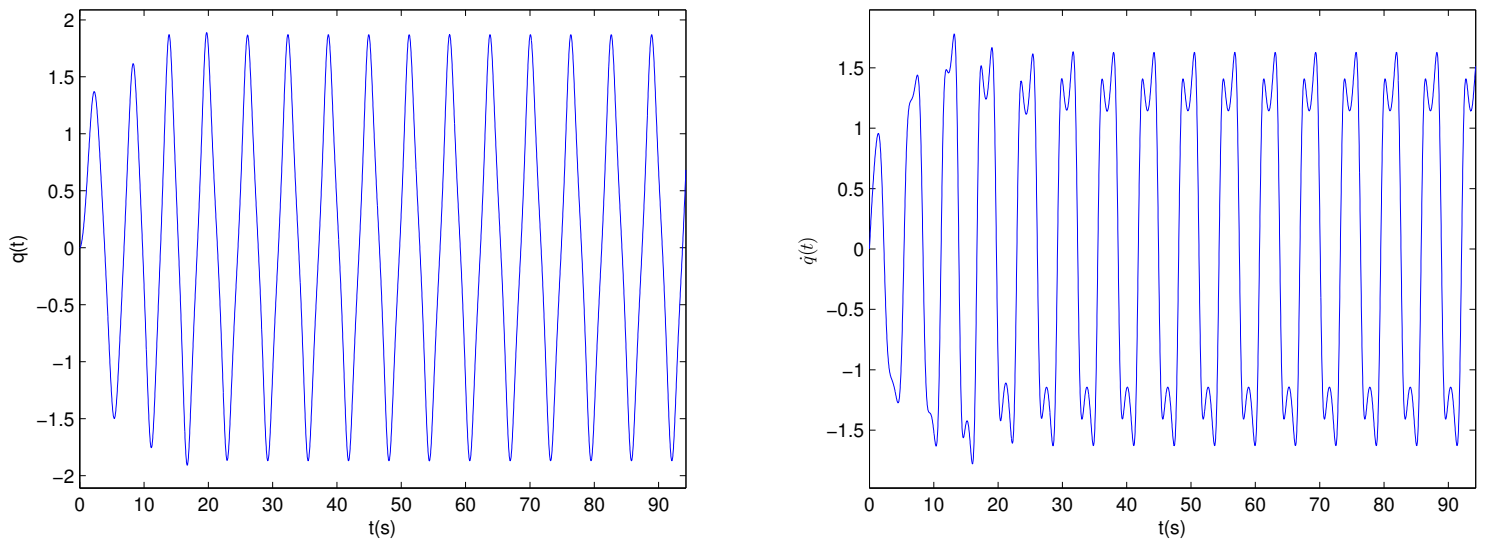

Figure 5: Case 2: displacement versus time(left), velocity versus time(right), $m=1, c=0.5, \omega=1.0, t_{0}=13 T, t=[0,23 T], d t=0.02$, $q(0)=\dot{q}(0)=0$
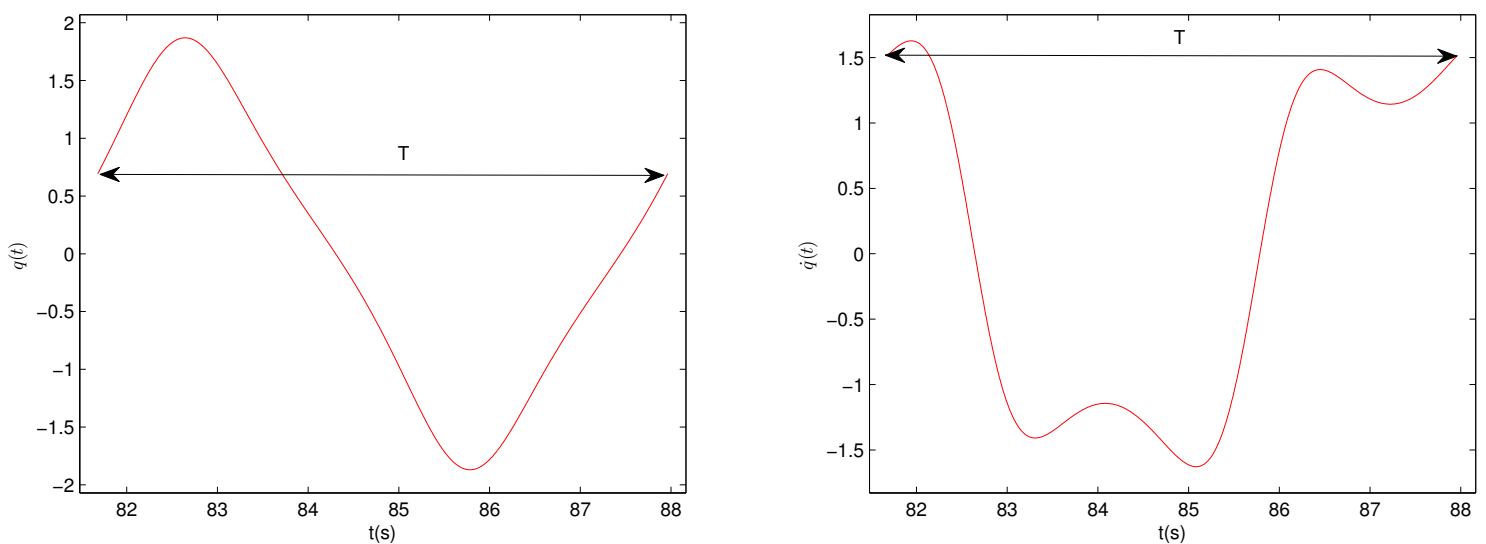

Figure 6: Case 2: displacement versus time(left), velocity versus time(right), $m=1, c=0.5, \omega=1.0, t_{0}=13 T, t=[0,23 T], d t=0.02$, $q(0)=\dot{q}(0)=0$ 

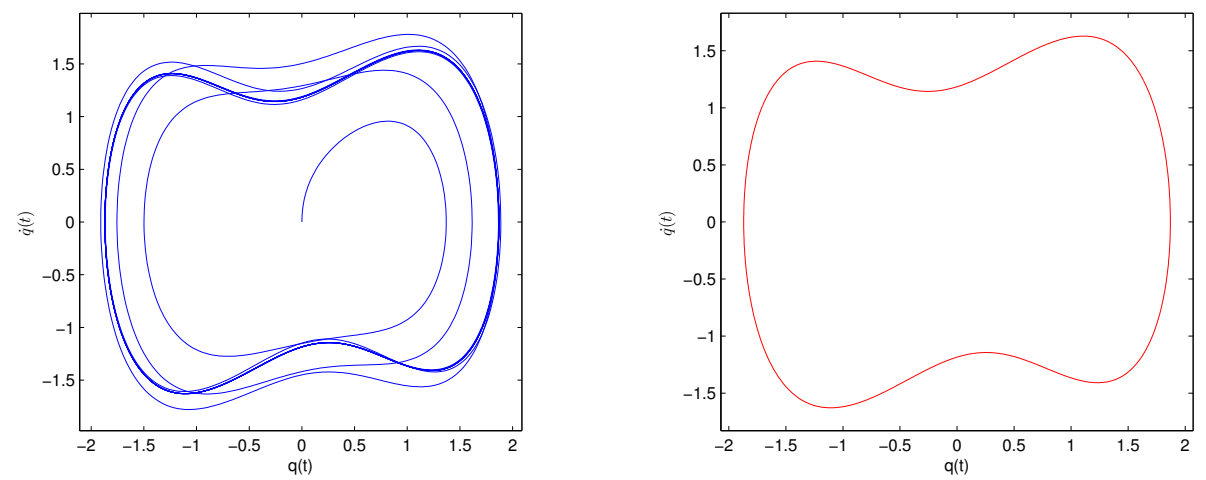

Figure 7: Case 2: orbits in phase space, $m=1, c=0.5, \omega=1.0, t_{0}=13 T, t=[0,23 T], d t=0.02, q(0)=\dot{q}(0)=0$

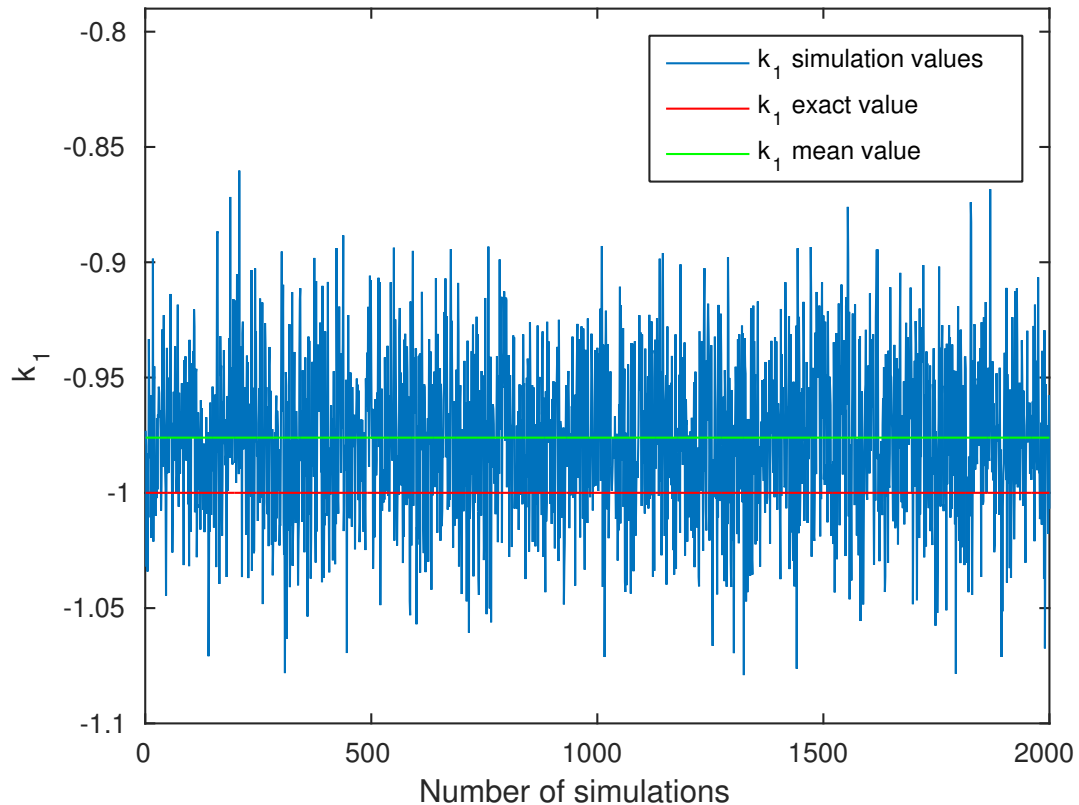

Figure 8: Case 2: $k_{1}$ simulation values at noise level 2.5\%, $m=1, c=0.5, \omega=1.0, t_{0}=13 T, t=[0,23 T], d t=0.02, q(0)=\dot{q}(0)=0$ 


\begin{tabular}{|c|c|c|c|c|c|c|}
\hline Number of simulations & \multicolumn{2}{|c|}{$\Gamma$} & \multicolumn{2}{|c|}{$k_{1}$} & \multicolumn{2}{|c|}{$k_{2}$} \\
\hline 2000 & \multicolumn{2}{|c|}{ Amplitude } & \multicolumn{2}{|c|}{ Linear coefficient } & \multicolumn{2}{|c|}{ Nonlinear coefficient } \\
\hline Exact & \multicolumn{2}{|c|}{2.0} & \multicolumn{2}{|c|}{1.0} & \multicolumn{2}{|c|}{0.2} \\
\hline Noise level & Numerical & Error(\%) & Numerical & Error(\%) & Numerical & Error(\%) \\
\hline \multirow[t]{6}{*}{$\begin{array}{c}\delta=0 \\
\delta=0.5 \%\end{array}$} & 1.9989 & $5.4932 e-2$ & 1.0021 & $2.1318 e-1$ & $1.9941 e-1$ & $2.9335 e-1$ \\
\hline & $\operatorname{Max}$ & $1.7675 e+1$ & Max & 4.1054 & Max & 2.9303 \\
\hline & Min & $3.8554 e-5$ & Min & $8.6371 e-4$ & Min & $1.8851 e-4$ \\
\hline & Mean & $5.5184 e-2$ & Mean & $9.1969 e-1$ & Mean & 0.65478 \\
\hline & $\sigma$ & $3.2772 e-2$ & $\sigma$ & $6.8277 e-1$ & $\sigma$ & 0.48683 \\
\hline & $5 \sigma$ & $2.1905 e-1$ & $5 \sigma$ & 4.3335 & $5 \sigma$ & 3.0889 \\
\hline \multicolumn{7}{|l|}{$\delta=1.0 \%$} \\
\hline & Max & $2.7177 e-1$ & Max & 9.8332 & Max & 6.5622 \\
\hline & Min & $7.7426 e-5$ & Min & $1.8461 e-3$ & Min & $4.8353 e-5$ \\
\hline & Mean & $6.7037 e-2$ & Mean & 1.8303 & Mean & 1.249 \\
\hline & $\sigma$ & $4.7218 e-2$ & $\sigma$ & 1.3781 & $\sigma$ & $9.44 e-1$ \\
\hline & $5 \sigma$ & $3.0312 e-1$ & $5 \sigma$ & 8.7208 & $5 \sigma$ & 5.969 \\
\hline \multicolumn{7}{|l|}{$\delta=1.5 \%$} \\
\hline & Max & $3.8822 e-1$ & $\operatorname{Max}$ & $1.222 e+1$ & $\operatorname{Max}$ & 8.4966 \\
\hline & Min & $4.4493 e-5$ & Min & $5.9917 e-3$ & Min & $2.4786 e-3$ \\
\hline & Mean & $8.8763 e-2$ & Mean & 2.8246 & Mean & 1.9298 \\
\hline & $\sigma$ & $6.6917 e-2$ & $\sigma$ & 2.059 & $\sigma$ & 1.3922 \\
\hline & $5 \sigma$ & $4.2335 e-1$ & $5 \sigma$ & $1.3119 e+1$ & $5 \sigma$ & 8.8907 \\
\hline \multicolumn{7}{|l|}{$\delta=2.0 \%$} \\
\hline & Max & $5.0268 e-1$ & Max & $1.6084 e+1$ & Max & $1.0613 e+1$ \\
\hline & Min & $1.1417 e-3$ & Min & $2.2156 e-3$ & Min & $1.7996 e-1$ \\
\hline & Mean & $1.1251 e-1$ & Mean & 3.7244 & Mean & 2.5463 \\
\hline & $\sigma$ & $8.6938 e-2$ & $\sigma$ & 2.8492 & $\sigma$ & 1.9284 \\
\hline & $5 \sigma$ & $5.472 e-1$ & $5 \sigma$ & $1.797 e+1$ & $5 \sigma$ & $1.2188 e+1$ \\
\hline \multicolumn{7}{|l|}{$\delta=2.5 \%$} \\
\hline & Max & $5.9335 e-1$ & Max & $2.0153 e+1$ & Max & $1.3395 e+1$ \\
\hline & Min & $1.4682 e-4$ & Min & $5.3766 e-3$ & Min & $2.1279 e-3$ \\
\hline & Mean & $1.418 e-1$ & Mean & 4.7327 & Mean & 3.2515 \\
\hline & $\sigma$ & $1.0793 e-1$ & $\sigma$ & 3.5931 & $\sigma$ & 2.4403 \\
\hline & $5 \sigma$ & $6.8143 e-1$ & $5 \sigma$ & $2.2698 e+1$ & $5 \sigma$ & $1.5453 e+1$ \\
\hline
\end{tabular}

Table 1: Case 1: Errors according to the different noise levels, $m=1, c=0.2, \omega=1.4, t_{0}=21 T, t=[0,23 T], d t=0.02, q(0)=\dot{q}(0)=0$ 


\begin{tabular}{|c|c|c|c|c|c|c|}
\hline Number of simulations & \multicolumn{2}{|c|}{$\Gamma$} & \multicolumn{2}{|c|}{$k_{1}$} & \multicolumn{2}{|c|}{$k_{2}$} \\
\hline 2000 & \multicolumn{2}{|c|}{ Amplitude } & \multicolumn{2}{|c|}{ Linear coefficient } & \multicolumn{2}{|c|}{ Nonlinear coefficient } \\
\hline Exact & \multicolumn{2}{|c|}{1.1} & \multicolumn{2}{|c|}{-1.0} & \multicolumn{2}{|c|}{1.0} \\
\hline Noise level & Numerical & Error $(\%)$ & Numerical & Error $(\%)$ & Numerical & Error(\%) \\
\hline \multirow[t]{6}{*}{$\begin{array}{c}\delta=0 \\
\delta=0.5 \%\end{array}$} & 1.1013 & $1.1628 e-1$ & $-9.9945 e-1$ & $5.4864 e-2$ & $9.9935 e-1$ & $6.4593 e-2$ \\
\hline & Max & $1.774 e-1$ & Max & 2.4499 & Max & 1.171 \\
\hline & Min & $5.4243 e-2$ & Min & $1.6796 e-4$ & Min & $1.2237 e-5$ \\
\hline & Mean & $1.1866 e-1$ & Mean & $5.6558 e-1$ & Mean & $2.6294 e-1$ \\
\hline & $\sigma$ & $1.9633 e-2$ & $\sigma$ & $4.0827 e-1$ & $\sigma$ & $1.9281 e-1$ \\
\hline & $5 \sigma$ & $2.1683 e-1$ & $5 \sigma$ & 2.6069 & $5 \sigma$ & 1.227 \\
\hline \multicolumn{7}{|l|}{$\delta=1.0 \%$} \\
\hline & $\operatorname{Max}$ & $2.5865 e-1$ & $\operatorname{Max}$ & 5.3898 & $\operatorname{Max}$ & 2.4047 \\
\hline & Min & $5.8067 e-3$ & Min & $1.0386 e-3$ & Min & $1.2484 e-4$ \\
\hline & Mean & $1.2858 e-1$ & Mean & 1.123 & Mean & $5.1704 e-1$ \\
\hline & $\sigma$ & $3.9666 e-2$ & $\sigma$ & $8.4979 e-1$ & $\sigma$ & $3.8348 e-1$ \\
\hline & $5 \sigma$ & $3.2691 e-1$ & $5 \sigma$ & 5.3719 & $5 \sigma$ & 2.4344 \\
\hline \multicolumn{7}{|l|}{$\delta=1.5 \%$} \\
\hline & $\operatorname{Max}$ & $3.209 e-1$ & $\operatorname{Max}$ & 7.8892 & Max & 3.6193 \\
\hline & Min & $9.0453 e-4$ & Min & $6.2911 e-4$ & Min & $9.8706 e-4$ \\
\hline & Mean & $1.3792 e-1$ & Mean & 1.7835 & Mean & $8.1135 e-1$ \\
\hline & $\sigma$ & $5.6846 e-2$ & $\sigma$ & 1.3373 & $\sigma$ & $6.0597 e-1$ \\
\hline & $5 \sigma$ & $4.2215 e-1$ & $5 \sigma$ & 8.4701 & $5 \sigma$ & 3.8412 \\
\hline \multicolumn{7}{|l|}{$\delta=2.0 \%$} \\
\hline & $\operatorname{Max}$ & $4.12093 e-1$ & Max & $1.1175 e+1$ & Max & 4.5748 \\
\hline & Min & $5.4512 e-4$ & Min & $2.0216 e-3$ & Min & $2.8116 e-3$ \\
\hline & Mean & $1.5525 e-1$ & Mean & 2.6298 & Mean & 1.2045 \\
\hline & $\sigma$ & $7.5874 e-2$ & $\sigma$ & 1.9432 & $\sigma$ & $8.7542 e-1$ \\
\hline & $5 \sigma$ & $5.3462 e-1$ & $5 \sigma$ & $1.2346 e+1$ & $5 \sigma$ & 5.5816 \\
\hline \multicolumn{7}{|l|}{$\delta=2.5 \%$} \\
\hline & $\operatorname{Max}$ & $5.7125 e-1$ & $\operatorname{Max}$ & $1.4762 e+1$ & Max & 6.5401 \\
\hline & Min & $1.3772 e-4$ & Min & $7.2127 e-4$ & Min & $2.1058 e-4$ \\
\hline & Mean & $1.8006 e-1$ & Mean & 3.4881 & Mean & 1.6099 \\
\hline & $\sigma$ & $9.4615 e-2$ & $\sigma$ & 2.5305 & $\sigma$ & 1.1529 \\
\hline & $5 \sigma$ & $6.5314 e-1$ & $5 \sigma$ & $1.6141 e+1$ & $5 \sigma$ & 7.3746 \\
\hline
\end{tabular}

Table 2: Case 2: Errors according to the different noise levels, $m=1, c=0.5, \omega=1.0, t_{0}=13 T, t=[0,15 T], d t=0.02, q(0)=\dot{q}(0)=0$ 


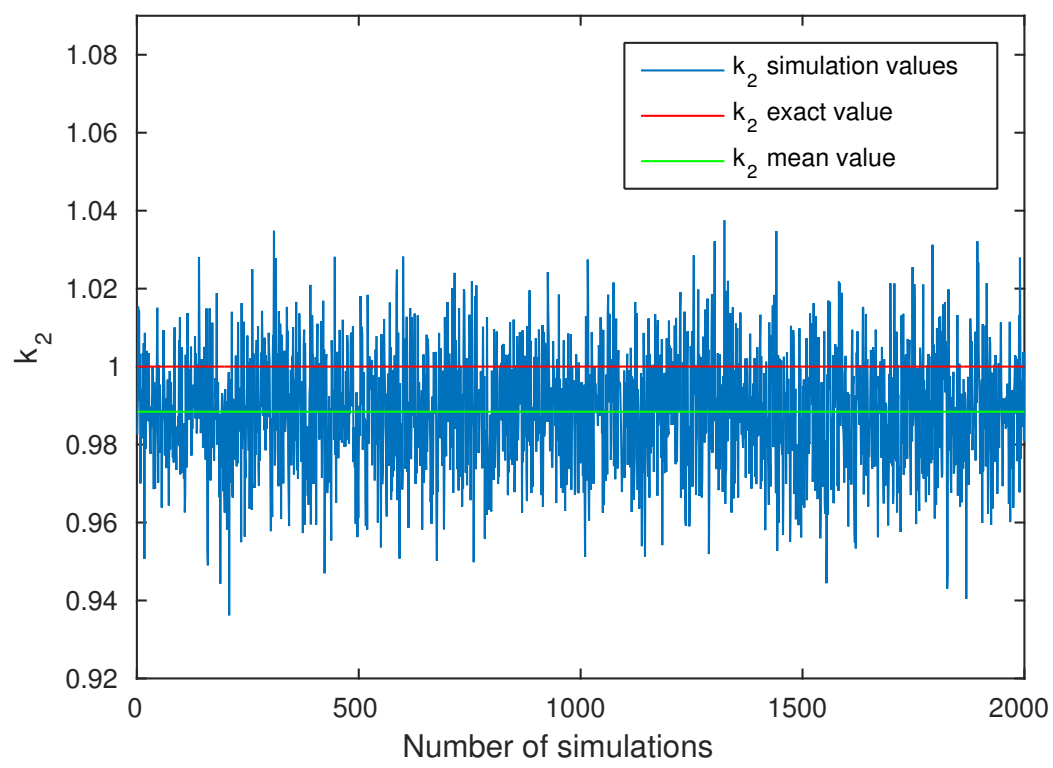

Figure 9: Case 2: $k_{2}$ simulation values at noise level 2.5\%, $m=1, c=0.5, \omega=1.0, t_{0}=13 T, t=[0,23 T], d t=0.02, q(0)=\dot{q}(0)=0$

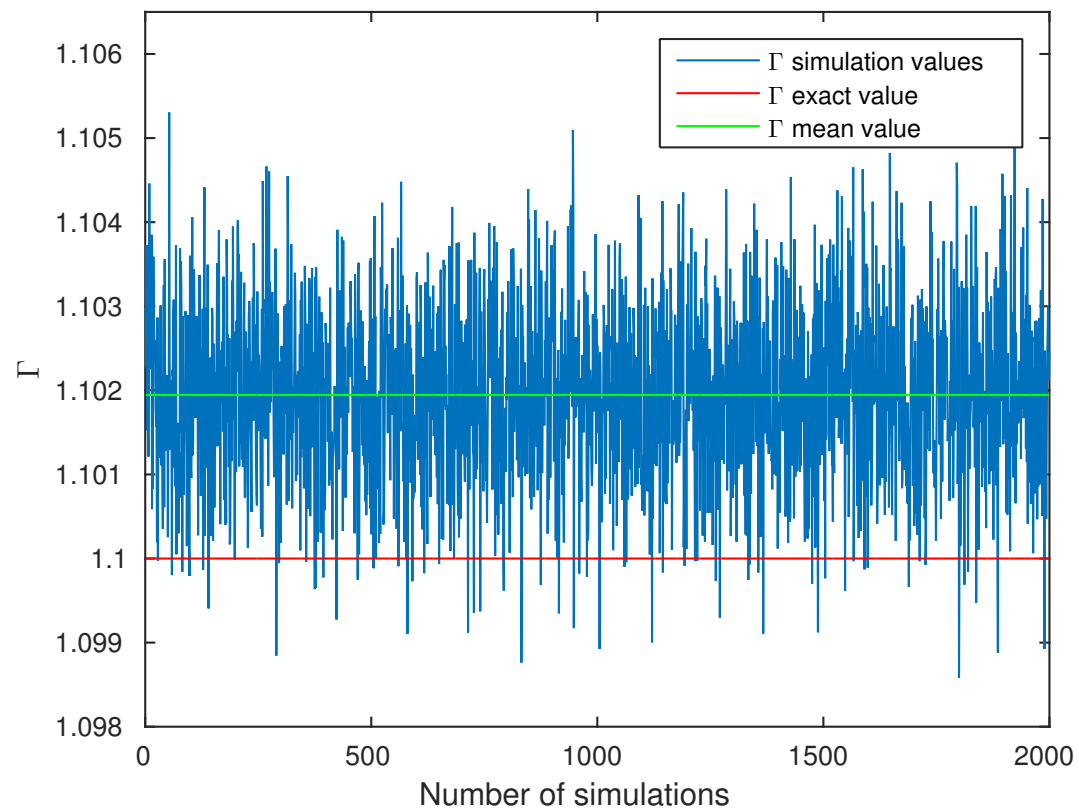

Figure 10: Case 2: $\Gamma$ simulation values at noise level 2.5\%, $m=1, c=0.5, \omega=1.0, t_{0}=13 T, t=[0,23 T], d t=0.02, q(0)=\dot{q}(0)=0$ 